\title{
Range-only based cooperative localization for mobile robots
}

\author{
Cyrille Pierre, Roland Chapuis, Romuald Aufrère \\ Institut Pascal \\ Clermont-Ferrand, France \\ \{cyrille.pierre, roland.chapuis, romuald.aufrere\}@uca.fr
}

\author{
Jean Laneurit, Christophe Debain \\ Irstea \\ Clermont-Ferrand, France \\ \{jean.laneurit, christophe.debain\}@irstea.fr
}

\begin{abstract}
In this paper, we address the problem of localizing mobile robots based on range-only measurements from low cost Ultra-Wide-Band (UWB) sensors. The proposed solution considers every static or mobile object as beacons with contextual information. A beacon-to-beacon measurement is performed using UWB sensors and the position estimation is computed by the target beacon. This strategy allows to hide the cooperative localization problem behind these measurements. The fusion algorithm is based on a Split Intersection Covariance Filter which allows to correctly handle the correlation between the poses estimations of the beacons. We present the consistency of this solution using a simulation with 3 robots and 4 static beacons and a real experimentation with 1 robot and 3 static beacons.
\end{abstract}

Keywords-cooperative localization, range only localization, UWB, Split Intersection Covariance, v2v localization, v2i localization

\section{INTRODUCTION}

Localization is a requirement in a lot of mobile robots application. A robot is able to locate itself using its own proprioceptive or exteroceptive sensors. However the quality of this localization will depend of the accuracy and the availability of these sensors. When several robots work in a same environment, the localization can be cooperative if the robots are able to detect each other and exchange their information. A solution can be to gather the sensors measurements of the robots and perform a centralized localization. This solution allows to keep the correlation between the robot state vectors but the algorithm cannot be performed in real time if the number of robot is high and this can also lead to high bandwidth usage. [1], [2] have proposed an approach to distribute the computation between the robots.

The modern cooperative localization solutions tend to focus on the decentralized approach which has better real-time performance but is suboptimal [3], [4]. If the robot pose estimations are computed independently, the correlation between these poses will be lost. A Kalman filter cannot correctly handle this problem because the robot pose estimations will be considered as fully independent and the estimation will be inconsistent. One of the solution is to avoid to reuse the same information several times. [5] propose an approach where each robot computes a local estimation based on its sensors and a fused estimation including all other robots informations. Only the local estimation is shared with other robots. Another solution [3] is based on the Covariance Intersection Filter
[6]. In this filter, the correlation between the measurements and state vector are considered as fully dependent. This corresponds to a pessimistic version of the Kalman Filter. This dependency can be configured more precisely with the Split Covariance Intersection Filter and allows to make better pose estimation [7], [8]. It is also possible to solve the problem using a set approach [9].

In most of these approaches, a measurement with another robot corresponds to a pose measurement. This type of measurement allows to exploit all the information of the state vector of the other robot. In practice, it is difficult to measure a pose difference. This can be done using LIDARs [10], [11] or camera [12] but these solutions requires data processing and impose constraints on the robots. The main problem mentioned in this paper is the ability to perform a cooperative localization with heterogeneous robots and with robot-to-robot or robotto-infrastructure measurements. The sensor able to make this measurements must be easily embedded in any object.

The Ultra Wide Band (UWB) sensor meets this constraint. It allows to make range measurement from another UWB sensor with a centimetric precision and it is low cost. However, the technology of this sensor does not allow to exploit the classic trilateration process because the range measurement requests cannot be done simultaneously [13], [14]. Moreover, it is well know that range only data lead to very non-linear functions that are difficult to handle for fusion.

In this paper, we propose an approach where every static or mobile objects are considered as beacons. These beacons can correspond to a mobile robot or a fixed object in the infrastructure but all these beacons must incorporate a UWB sensor to perform range measurement with other beacons (Fig. 1). A measurement with a beacon involve the range measurement but also the current position estimation (but not the orientation) computed by the target beacon. With this generic architecture, the cooperative localization aspect is hidden behind these measurements. A robot does not need to know if the target is a robot or an element of the infrastructure. This strategy simplifies the cooperative localization problem but it does not solve it. Suppose a static beacon has a nonnegligible position uncertainty. A robot should make several measurements from this beacon, but this induces an overuse of the position information of the beacon. A Kalman filter cannot correctly handle this highly correlated position uncertainty 


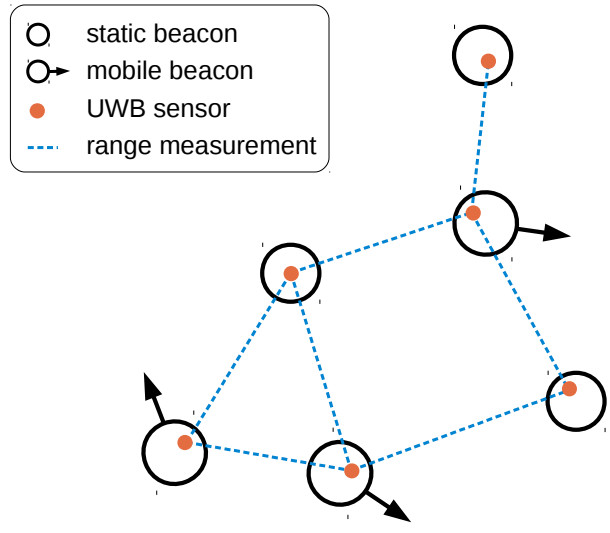

Fig. 1. Illustration of the beacons (robots or static object) and the range measurements of its UWB sensors

because it will introduce inconsistency in the robot state estimation. Our solution is based on the Split Covariance Intersection filter which allows to split the covariance matrix into independent and dependent covariance matrices [6], [15]. In our case, the range measurement of a beacon can be considered independent of the previous measurement but its position is very correlated to the previous one.

\section{LOCALIZATION ALGORITHM}

From a general point of view, this algorithm acts as cooperative localization but for a robot, this is a classical fusion algorithm with simple robots measurements. In this approach, the localization of each robot is computed individually, thus the algorithm described here will only concern the current robot. However, the same algorithm is applied to every other robots or beacons if needed. The localization problem is modeled by an estimation of the state $\mathbf{x}_{k}$ of the robot at the moment $k$. We use a bayesian approach based on the classical state model:

$$
\begin{aligned}
\mathbf{x}_{k} & =f\left(\mathbf{x}_{k-1}, \mathbf{u}_{k}, \mathbf{w}_{k}\right) \\
\mathbf{z}_{k}^{(i)} & =h^{(i)}\left(\mathbf{x}_{k}, \mathbf{v}_{k}^{(i)}\right)
\end{aligned}
$$

The evolution function $f$ is based on the vector $\mathbf{u}_{k}$ including proprioceptive information of the robot and the involved noise vector $\mathbf{w}_{k}$ of the model. The function $h^{(i)}$ describes one of the observation model. This function allows to link the $i$ th measurement vector $\mathbf{z}_{k}^{(i)}$ to the robot state $\mathbf{x}_{k}$. This model also involves a noise vector $\mathbf{v}_{k}^{(i)}$.

\section{A. Split Intersection Covariance Filter}

The Split Intersection Covariance Filter (SCIF) is an alternative between the Kalman Filter (KF) and the Covariance Intersection Filter (CIF) [6] . The KF allows to estimate a dynamic state by merging informations from several sources. One of the particularity of this algorithm is to consider that every information is independent. Conversely, the CIF considers that every information is potentialy correlated to another. The SCIF combines these two approaches to provide more flexibility in the control of the information correlation.
In a standard KF, the estimate of a state $\mathbf{x}_{k}$ at the moment $k$ corresponds to a pair $\left(\mathbf{x}_{k \mid k}, \mathbf{P}_{k \mid k}\right)$ where $\mathbf{x}_{k \mid k}$ is the state vector and $\mathbf{P}_{k \mid k}$ is its associated covariance matrix.

$$
p\left(\mathbf{x}_{k}\right) \sim \mathcal{N}\left(\mathbf{x}_{k \mid k}, \mathbf{P}_{k \mid k}\right)
$$

In a SCIF, this matrix is cut in two parts: the independent covariance matrix $\mathbf{P}_{i, k \mid k}$ corresponding to the covariance of the state without correlation from any sources and the dependent covariance matrix $\mathbf{P}_{d, k \mid k}$ corresponding to the correlated one.

$$
\mathbf{P}_{k \mid k}=\mathbf{P}_{i, k \mid k}+\mathbf{P}_{d, k \mid k}
$$

Thus the SCIF works with the triplet $\left(\mathbf{x}_{k \mid k}, \mathbf{P}_{i, k \mid k}, \mathbf{P}_{d, k \mid k}\right)$. This formalism is also applied to any information vector to fuse.

Given the triplet $\left(\mathbf{u}_{k}, \mathbf{Q}_{i k}, \mathbf{Q}_{d k}\right)$ of proprioceptive information, the non-linear prediction steps of the SCIF are:

$$
\begin{aligned}
\mathbf{x}_{k \mid k-1} & =f\left(\mathbf{x}_{k-1 \mid k-1}, \mathbf{u}_{k}, \mathbf{0}\right) \\
\mathbf{P}_{k \mid k-1} & =\mathbf{J}_{f x} \mathbf{P}_{k-1 \mid k-1} \mathbf{J}_{f x}^{T}+\mathbf{J}_{f w} \mathbf{Q}_{k} \mathbf{J}_{f w}^{T} \\
\mathbf{P}_{i, k \mid k-1} & =\mathbf{J}_{f x} \mathbf{P}_{i, k-1 \mid k-1} \mathbf{J}_{f x}^{T}+\mathbf{J}_{f w} \mathbf{Q}_{i k} \mathbf{J}_{f w}^{T} \\
\mathbf{P}_{d, k \mid k-1} & =\mathbf{P}_{k \mid k-1}-\mathbf{P}_{i, k \mid k-1}
\end{aligned}
$$

with:

$$
\mathbf{J}_{f x}=\frac{\partial f}{\partial \mathbf{x}}\left(\mathbf{x}_{k-1 \mid k-1}, \mathbf{u}_{k}, \mathbf{0}\right) \quad \mathbf{J}_{f w}=\frac{\partial f}{\partial \mathbf{w}}\left(\mathbf{x}_{k-1 \mid k-1}, \mathbf{u}_{k}, \mathbf{0}\right)
$$

For an observation function $h$ and a measurement $\left(\mathbf{z}_{k}, \mathbf{R}_{i k}, \mathbf{R}_{d k}\right)$, the update steps of the SCIF are:

$$
\begin{aligned}
\mathbf{P}_{1}= & \omega^{-1} \mathbf{P}_{d, k \mid k-1}+\mathbf{P}_{i, k \mid k-1} \\
\mathbf{P}_{2}= & (1-\omega)^{-1} \mathbf{R}_{d k}+\mathbf{R}_{i k} \\
\mathbf{K}= & \mathbf{P}_{1} \mathbf{J}_{h x}^{T}\left(\mathbf{J}_{h x} \mathbf{P}_{1} \mathbf{J}_{h x}^{T}+\mathbf{J}_{h v} \mathbf{P}_{2} \mathbf{J}_{h v}^{T}\right)^{-1} \\
\mathbf{x}_{k \mid k}= & \mathbf{x}_{k \mid k-1}+\mathbf{K}\left(\mathbf{z}_{k}-\mathbf{h}\left(\mathbf{x}_{k \mid k-1}, 0\right)\right) \\
\mathbf{P}_{k \mid k}= & \left(\mathbf{I}-\mathbf{K} \mathbf{J}_{h x}\right) \mathbf{P}_{1} \\
\mathbf{P}_{i, k \mid k}= & \left(\mathbf{I}-\mathbf{K} \mathbf{J}_{h x}\right) \mathbf{P}_{i, k \mid k-1}\left(\mathbf{I}-\mathbf{K} \mathbf{J}_{h x}\right)^{T} \\
& +\left(\mathbf{K} \mathbf{J}_{h v}\right) \mathbf{R}_{i k}\left(\mathbf{K} \mathbf{J}_{h v}\right)^{T} \\
\mathbf{P}_{d, k \mid k}= & \mathbf{P}_{k \mid k}-\mathbf{P}_{i, k \mid k}
\end{aligned}
$$

with:

$$
\mathbf{J}_{h x}=\frac{\partial h}{\partial \mathbf{x}}\left(\mathbf{x}_{k \mid k-1}, \mathbf{0}\right) \quad \mathbf{J}_{h v}=\frac{\partial h}{\partial \mathbf{u}}\left(\mathbf{x}_{k \mid k-1}, \mathbf{0}\right)
$$

In the (10) and 11, the independent part of the covariance matrices are used as in a KF and the dependent part as a CIF. The coefficient $\omega \in[0,1]$ is choosen to minimize the determinant of the resulting covariance matrix. It is equivalent to find the minimum volume of the hyper-ellipsoïd representing this covariance.

\section{B. The evolution model}

The robot state $\mathbf{x}$ is a vector containing the position $x, y, z$ and the orientation $\theta$ of the robot at the moment $k$.

$$
\mathbf{x}_{k}=\left[\begin{array}{llll}
x & y & z & \theta
\end{array}\right]_{k}^{T}
$$

The third geometric dimension is necessary because the UWB sensors can be placed to any height from ground and this height will affect the range measurement. However all robots 
move on a relatively flat ground, thus only the yaw angle is used for the orientation of the robot.

There are various robot models but, in this paper, only the Ackermann model has been used. The proprioceptive informations of our robots corresponds to the speed $s$ and the steering angle $\varphi$ of the front wheels. These measurements are associated to the noise vector $\mathbf{w}$.

$$
\mathbf{u}_{k}=\left[\begin{array}{ll}
s & \varphi
\end{array}\right]_{k}^{T} \quad \mathbf{w}_{k}=\left[\begin{array}{ll}
w_{s} & w_{\varphi}
\end{array}\right]_{k}^{T}
$$

Given the measurement period $\delta t$ and the wheelbase of the robot $L$, the evolution function is:

$$
f\left(\mathbf{x}_{k}, \mathbf{u}_{k}, \mathbf{w}_{k}\right)=\mathbf{x}_{k}+\left[\begin{array}{c}
\left(s+w_{s}\right) \delta t \cos \theta \\
\left(s+w_{s}\right) \delta t \sin \theta \\
0 \\
-\frac{s+w_{s}}{L} \delta t \tan \left(\varphi+w_{\varphi}\right)
\end{array}\right]_{k}
$$

\section{The observation models}

Different observation models based on different sensors are used in this approach but the main model rely the UWB sensors of beacons. These sensors can perform range-only measurement $\mathbf{z}_{b k}$ at the moment $k$ but we add some contextual informations: the position of the target UWB and the uncertainty of this position. This uncertainty follows a normal distribution and can be modeled as a mean value $\mathbf{b}_{k}$ and covariance matrix $\mathbf{B}_{k}$. If the target beacon is static, its position have been fixed at its initialization. But if this beacon corresponds to a mobile object (like another robot), its position information is extracted from its current state estimation. In both cases, a measurement at the moment $k$ will be correlated to previous measurements with the same target because its position information may change a little. This implies that the uncertainty of the target position must be processed as a dependent covariance. The Fig. 2 illustrates the importance of this configuration. If the only information source of the robot localization is an unique beacon, the position estimation of the robot cannot be better than the beacon position estimation.

Let $\left(\mathbf{z}_{b}, \mathbf{R}_{i}, \mathbf{R}_{d}\right)$ be a range measurement with a target beacon. The noise vector $\mathbf{v}$ of this measurement contains the range measurement noise $v_{r}$ and also the beacon position noise vector $\mathbf{v}_{p} \in \mathbb{R}^{3}$. Thus, $\mathbf{z}_{b}$ is a scalar corresponding to the range measurement.

$$
\mathbf{R}_{i}=\left[\begin{array}{cc}
\sigma_{r}^{2} & \mathbf{0} \\
\mathbf{0} & \mathbf{0}
\end{array}\right] \quad \mathbf{R}_{d}=\left[\begin{array}{ll}
0 & \mathbf{0} \\
\mathbf{0} & \mathbf{B}
\end{array}\right]
$$

$\mathbf{R}_{i}, \mathbf{R}_{d}$ contain the range measurement variance $\sigma_{r}^{2}$ and the position covariance matrix of the beacon $\mathbf{B}$. For a UWB sensor, the range measurement have a static error but the noise is white. The observation function $h_{b}$ of a range measurement uses the position of the target beacon $\mathbf{p}_{b}$ as a constant. Because the sensor of the robot used in this measurement can be placed

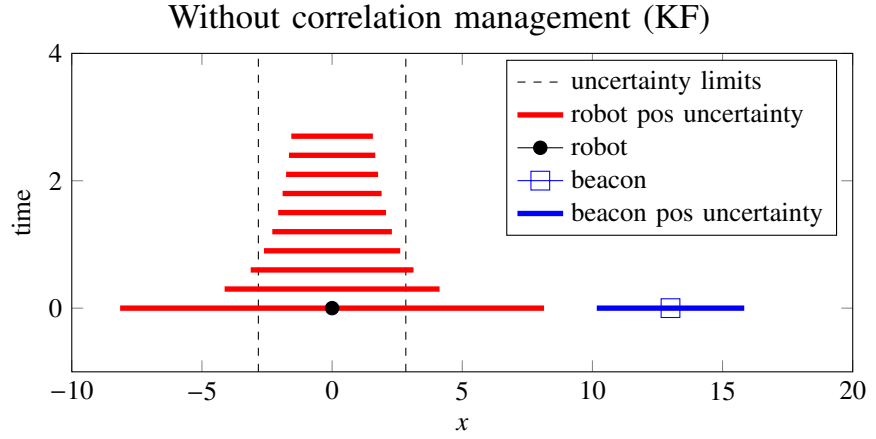

With correlation management (SCIF)

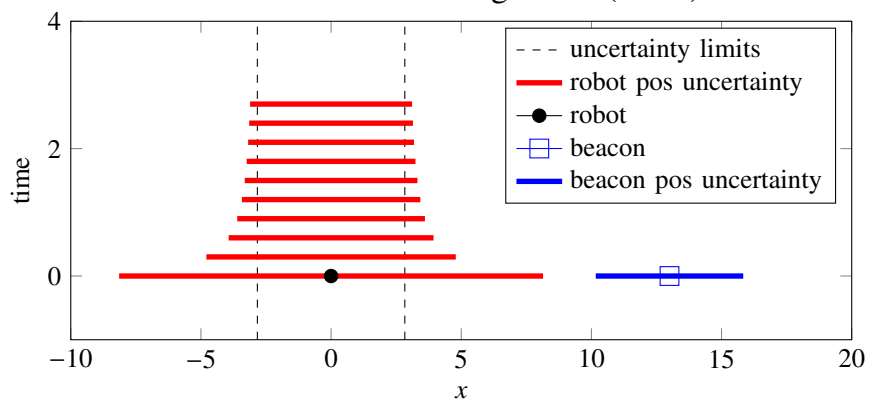

Fig. 2. Evolution of the robot position uncertainty after several noisy range measurements from a beacon. With a standard KF, the position uncertainty of the beacon is used as a new information for each range measurement, thus the robot position estimation will lose its integrity. In the second case, the position uncertainty is considered as fully dependent on the robot state, thus the overconvergence is avoided.

anywhere on the robot, this function also integrates a sensor position vector $\mathbf{p}_{s}$.

$$
\begin{aligned}
h_{b}(\mathbf{x}, \mathbf{v}) & =\left\|(x, y, z)^{T}+\operatorname{rot}(\theta) \mathbf{p}_{s}-\mathbf{p}_{b}-\mathbf{v}_{p}\right\|+v_{r} \\
\operatorname{rot}(\theta) & =\left[\begin{array}{ccc}
\cos \theta & -\sin \theta & 0 \\
\sin \theta & \cos \theta & 0 \\
0 & 0 & 1
\end{array}\right]
\end{aligned}
$$

A localization based on range measurement from individual beacon is unstable when the position uncertainty is significant because of the non-linearity of the model. That's why we use low cost GPS to make a brieve estimation of the robot position. Let $\left(\mathbf{z}_{g}, \mathbf{G}_{i}, \mathbf{G}_{d}\right)$ be a position measurement from a GPS, the vector $\mathbf{z}_{g} \in \mathbb{R}^{3}$ contains the measured position and $\mathbf{v}_{g}$ corresponds to the noise of this measurement. The measurements of this kind of sensor are easily disturbed by the environment and its noise cannot be considered as white. It is possible to improve the model and integrate a bias parameter in the robot state to model more accurately its noise [16]. Because we do not need to have an accurate model of this sensor, we choose to take into account this correlation by applying to the total covariance $\mathbf{G}$, a constant $\alpha \in[0 ; 1]$ to configure the dependencies of its covariance matrix.

$$
\mathbf{G}_{d}=\alpha \mathbf{G} \quad \mathbf{G}_{i}=(1-\alpha) \mathbf{G}
$$

This allows to limit the convergence of the estimation of the robot position. Given the sensor position vector $\mathbf{p}_{g}$, the 


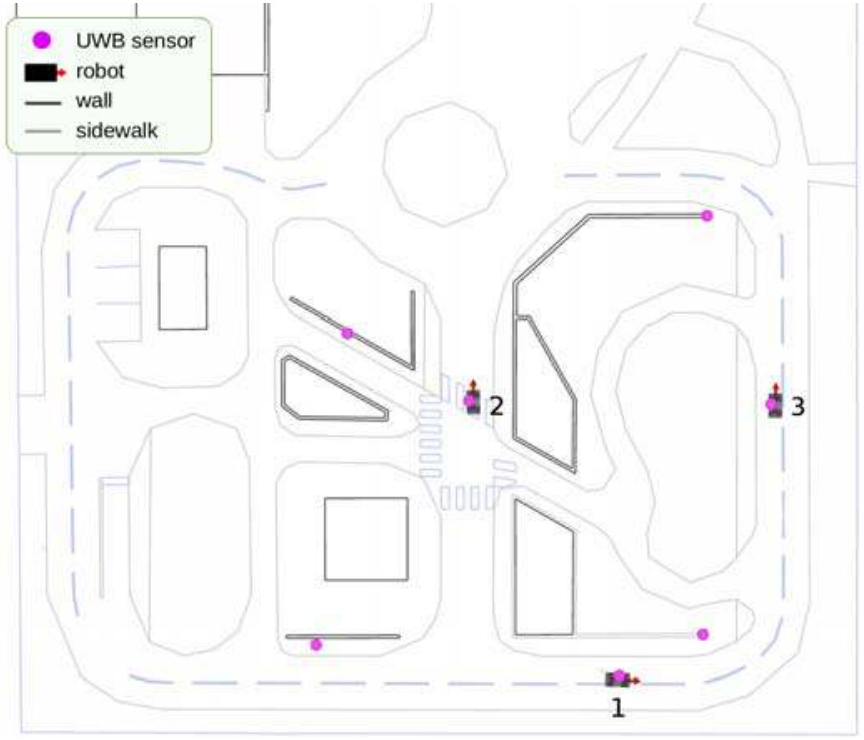

Fig. 3. A picture of the $57 \mathrm{~m} \times 51 \mathrm{~m}$ field with the initial position of the robots $(2.54 \mathrm{~m} \times 1.59 \mathrm{~m})$ and the static beacons fixed on the walls.

observation function is:

$$
h_{g}\left(\mathbf{x}, \mathbf{v}_{g}\right)=(x, y, z)^{T}+\operatorname{rot}(\theta) \mathbf{p}_{g}+\mathbf{v}_{g}
$$

A basic GPS sensor cannot make a good estimation of the altitude. Furthermore, the position of the beacons does not allows to improve the accuracy of the $z$ axis because this requires to place them very high. To solve this problem we exploit the fact that all robots move on the ground. Considering that the ground is relatively flat, we introduce a virtual sensor able to make direct measurements of the altitude. In a measurement $\left(\mathbf{z}_{m}, \mathbf{M}_{i}, \mathbf{M}_{d}\right)$, the scalar $\mathbf{z}_{m}$ corresponds to a measurement of the $z$ position. This sensor will send measurement periodically but it's always the same information, so the dependent part of the covariance matrix corresponds to the total covariance matrix $\mathbf{M}$ :

$$
\mathbf{M}_{d}=\mathbf{M} \quad \mathbf{M}_{i}=\mathbf{0}
$$

Let $\mathbf{v}_{m} \in \mathbb{R}$ denotes the measurement noise, the obervation function is:

$$
h_{m}\left(\mathbf{x}, \mathbf{v}_{m}\right)=z+\mathbf{v}_{m}
$$

\section{Simulation}

In this section, we present a simulation of our approach with 7 beacons : 3 mobile robots and 4 static UWB tags. All these objects are placed in a 3D simultaion environment of our real Pavin site iwhich contains walls and roads (Fig. $3,4)$. The robots move on this road and try to localize themselves using odometry, GPS and beacons measurements. But in this simulation, one of these robots is used as an accurately localized mobile beacon. The GPS used in this robot is able to make almost perfect position measurements. With this very low position uncertainty, the robot does not need to perform beacons' measurements to improve its localization.

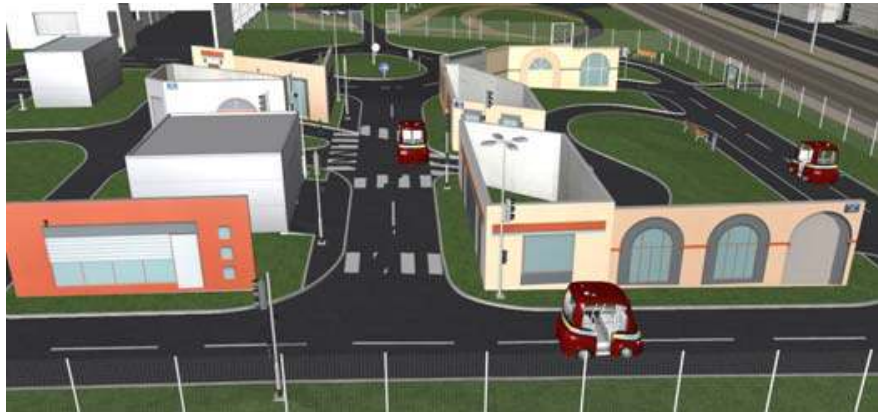

Fig. 4. The 3D environment of the simulator (4D-Virtualiz).

\begin{tabular}{|c|c|c|}
\hline \multicolumn{3}{|l|}{ Robot 1} \\
\hline Parameter & Value & Unit \\
\hline odometry measurement freq. & 10 & $\mathrm{~Hz}$ \\
\hline std. dev. of linear speed & 0.1 & $\mathrm{~ms}^{-1}$ \\
\hline std. dev. of steering angle & 0.01 & $\mathrm{rad}$ \\
\hline GPS measurement freq. & 10 & $\mathrm{~Hz}$ \\
\hline std. dev. of GPS $(x \& y)$ & 0.05 & $\mathrm{~m}$ \\
\hline std. dev. of GPS $(z)$ & 0.1 & $\mathrm{~m}$ \\
\hline beacons measurement freq. & 10 & $\mathrm{~Hz}$ \\
\hline \multicolumn{3}{|l|}{ Robot $2 \& 3$} \\
\hline odometry measurement freq. & 10 & $\mathrm{~Hz}$ \\
\hline std. dev. of linear speed & 0.2 & $\mathrm{~ms}^{-1}$ \\
\hline std. dev. of steering angle & 0.02 & rad \\
\hline GPS measurement freq. & 10 & $\mathrm{~Hz}$ \\
\hline std. dev. of GPS $(x \& y)$ & 12 & $\mathrm{~m}$ \\
\hline std. dev. of GPS $(z)$ & 20 & $\mathrm{~m}$ \\
\hline beacons measurement freq. & 10 & $\mathrm{~Hz}$ \\
\hline \multicolumn{3}{|l|}{ Static beacons } \\
\hline maximal range & 20 & $\mathrm{~m}$ \\
\hline std. dev. of position $(x, y \& z)$ & 0.28 & $\mathrm{~m}$ \\
\hline std. dev. of range measurement & 0.15 & $\mathrm{~m}$ \\
\hline
\end{tabular}

TABLE I

CONFIGURATION OF THE SIMULATION

The orientation estimation is computed by fusing odometry and low cost GPS measurements. The other two robots use GPS with simulated noises compatible with real data. The quality of the position estimation of these sensors are not sufficient to make efficient localization but there allows to make a brieve estimation at the begining of the simulation. The localization will be improved afterward by the beacons' range measurements. However, these measurements are subject to a simulated centered gaussian white noise. The Table I shows the configuration of the simulation.

During the simulation, each robot moves forward following its own trajectory. All the state vectors of the robots are initialized to the center of the area and with a sufficiently high uncertainty to cover all the area. The first GPS measurements and the odometry allow to position roughly the robot and correct its orientation. Beacons measurements start after 12 seconds of simulation. The robot will make measurement requests to beacons one after another but some of these requests will fail because of the maximal range $(20 \mathrm{~m})$ of the UWB sensors. Some areas does not allow to see more than one beacon if there is not other robot around.

The objective of this simulation is to validate the consistency of the pose estimation of the robots 2 and 3 . The quality 

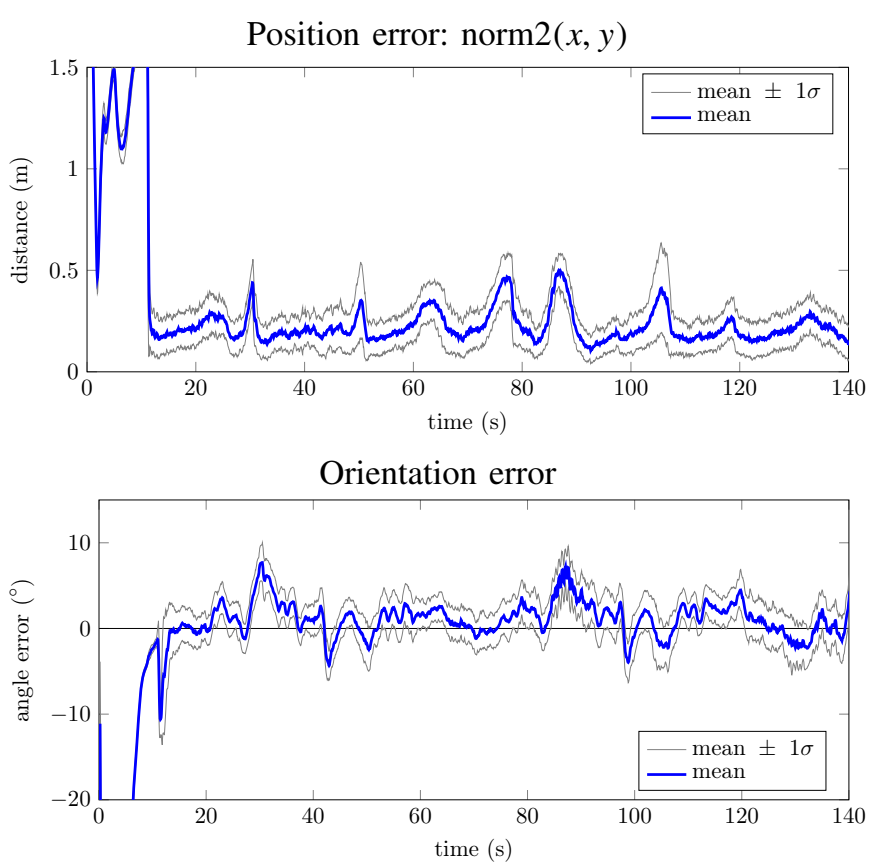

Mahalanobis distance of the state vector

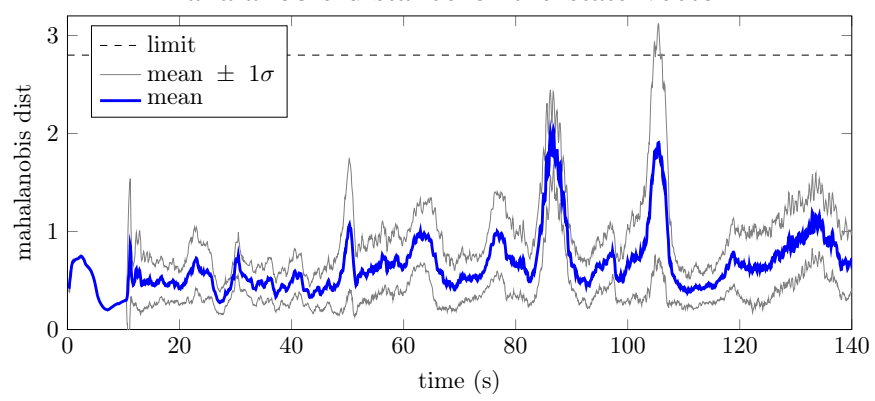

Fig. 5. The results of the localization of the robots 2 . The curves correspond to the mean error of the pose estimation and its standard deviation for 100 simulations.

of the localization is measured through the distance between the estimated pose of a robot and the real pose. A Monte Carlo procedure based on 100 simulations has been used to improve the results. The mean and the standard deviation of this simulations are shown in Fig. 5 and 6. During the first 12 seconds, the position error is significant because the GPS measurements are biased. However the high uncertainty of these measurements allows to keep a consistent estimation of the pose. When the beacons measurements start, the pose estimation became more accurate. During this period, the mean error of the position estimation of the robot 2 is $22.3 \mathrm{~cm}$ and $25.2 \mathrm{~cm}$ for the robot 3 . The root mean square of the orientation error is $2.26^{\circ}$ for the robot 2 and $4.65^{\circ}$ for the robot 3. The estimation is quite accurate taking account of the size of the field.

The mahalanobis distance betwwen the estimated triplet $(x, y, \theta)$ and the ground truth allows to evaluate the consistency of the localization. For a 3 degrees of freedom chi-square random variable, the square root of the $5 \%$ tail point is:
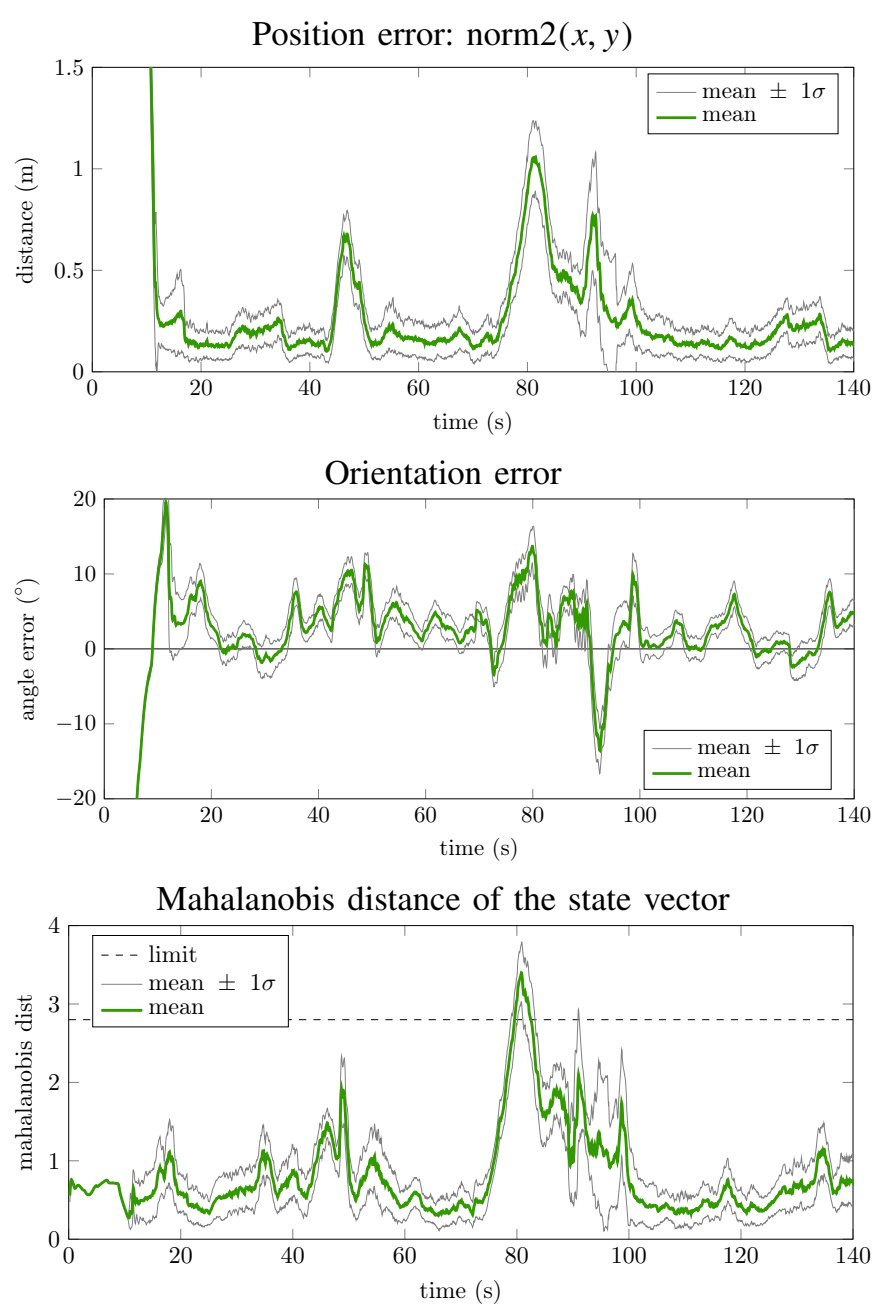

Fig. 6. The results of the localization of the robots 3 . The curves correspond to the mean error of the pose estimation and its standard deviation for 100 simulations.

$\sqrt{\chi_{3}^{2}(0.95)}=2.8$ Because the mahalanobis distance is lower than 2.8 more than $95 \%$ of the time, we consider that the result of the simulation is consistent for both robots 2 and 3 [17]. However we can see that, sometimes, the accuracy of robot's position decrease. This occured when the robot is not able to see more than one beacon. Consequently, the odometry noise cannot be corrected in all direction and the non-linearity of the range measurement pull the pose estimation off the ground truth.

\section{REAL EXPERIMENTATION}

In this section, we present an experimentation with a real robot. The environment is similar to the simulated one but the beacons configuration is different. The objective of this experimentation is to validate the algorithm with one robot and three static beacons under real-world conditions. The Fig. 7 shows this configuration. The robot is equiped with two GPS: a basic one and a RTK. The GPS RTK is used as ground truth and the basic GPS allows to make an estimation of the position at the begining of the experimentation. The beacons 


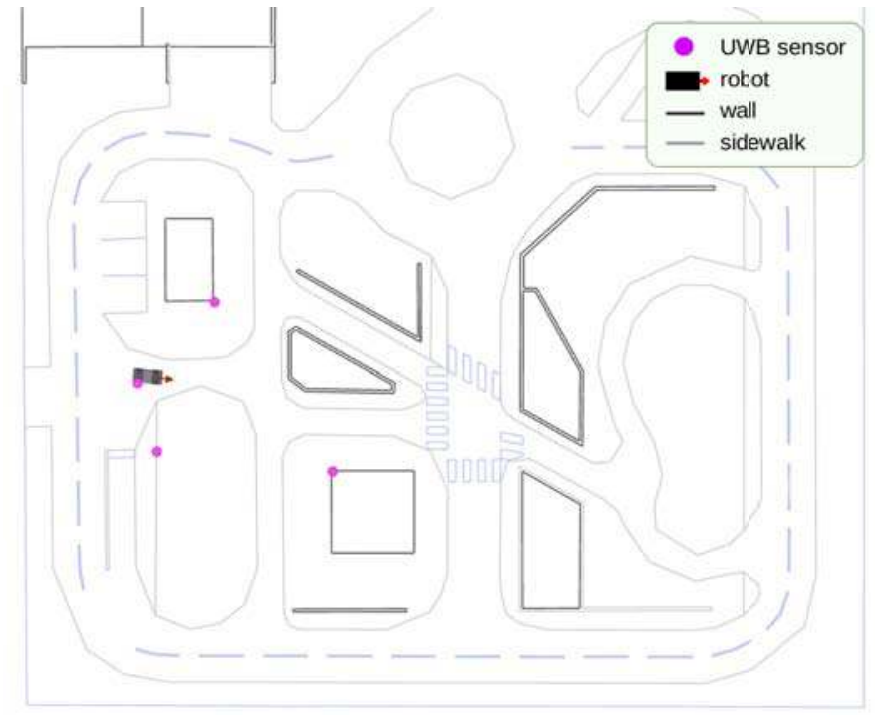

Fig. 7. The $57 \mathrm{~m} \times 51 \mathrm{~m}$ field with the initial position of the robots and the static beacons fixed on the walls

TABLE II

CONFIGURATION OF THE EXPERIMENTATION

\begin{tabular}{|l|c|l|}
\hline \multicolumn{3}{|c|}{ Robot } \\
\hline Parameter & Value & Unit \\
\hline odometry measurement freq. & 100 & $\mathrm{~Hz}$ \\
std. dev. of linear speed & 0.1 & $\mathrm{~m} \mathrm{~s}^{-1}$ \\
std. dev. of steering angle & 0.02 & $\mathrm{rad}$ \\
GPS measurement freq. & 5 & $\mathrm{~Hz}$ \\
std. dev. of GPS $(x \& y)$ & 10 & $\mathrm{~m}$ \\
std. dev. of GPS $(z)$ & 20 & $\mathrm{~m}$ \\
beacons measurement freq. & 10 & $\mathrm{~Hz}$ \\
\hline \multicolumn{2}{|c|}{ Static beacons } \\
\hline maximal range of position $(x, y \& z)$ & 25 & $\mathrm{~m}$ \\
std. dev. of $y 16$ & $\mathrm{~m}$ \\
std. dev. of range measurement & 0.15 & $\mathrm{~m}$ \\
\hline
\end{tabular}

measurements start after a short time to allow the robot to precise the orientation estimation before these measurements. Table II shows the configuration of experimentation.

The results of the experimentation are shown in Fig. 8. The mean error of the position estimation is $0.761 \mathrm{~m}$ and the root mean square error of the orientation is $5.97^{\circ}$. The position error is lower than this mean when the robot is able to see more than one beacon. If this is not the case, this error can increase depending of the position of the beacon relative to the robot. The most significant geometric error of the pose estimation occurs during the seconds 67-79. The robot can only use the GPS and odometry but the initial orientation error during this period causes a drift of the estimation.

The mahalanobis distance associated to the triplet $(x, y, \theta)$ is more significant than the one computed in the simulation but only $2.25 \%$ exceed the previously defined chi-square threshold. Thus, the localization is consistent. Some of the peaks of the mahalanobis distance come from significant range measurement error. The noise of these measurements is considered white but sometimes, the radio signal can bounce on the wall and the sensor will give us a wrong range. This
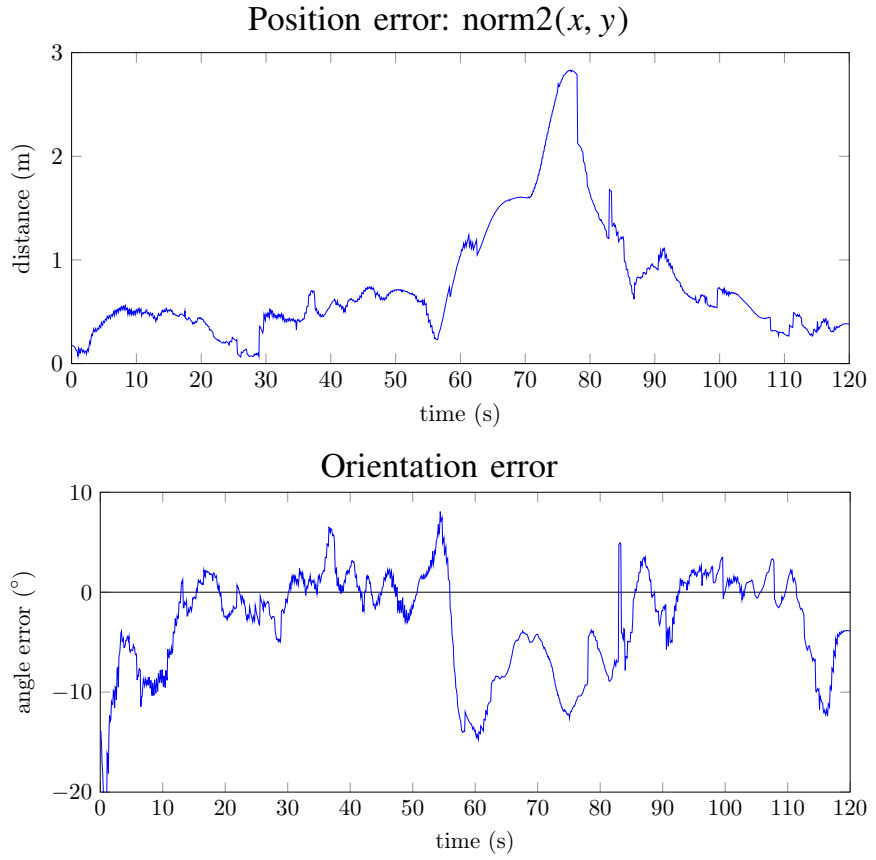

Mahalanobis distance of the state vector

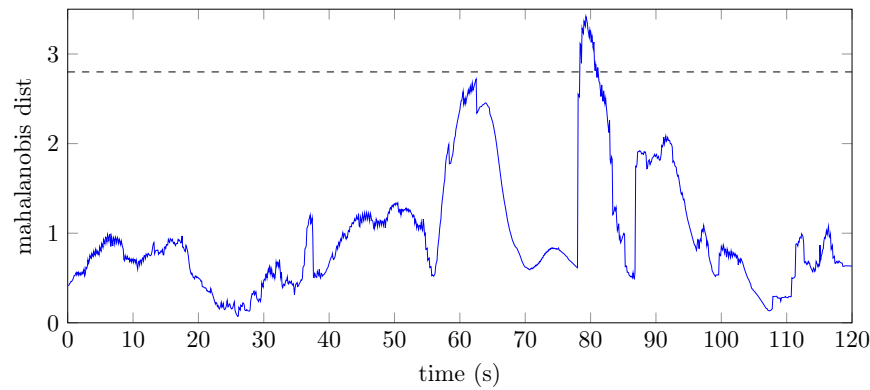

Fig. 8. The results of the localization of the robot in the experimentation The curves correspond to the error of the pose estimation with the real pose.

problem is known as the multipath effect [18]. To avoid this problem, we only take into account a range measurement if the mahalanobis distance between the position estimation before and after the measurement is lower than a threshold $(0.7$ in our case). This solution allows to eliminate the largest range error.

\section{CONCLUSION AND FUTURE DIRECTIONS}

In this paper we presented a localization algorithm based on range measurements from mobile or static beacons. If this algorithm is performed by several beacons, the localization becomes cooperative because of the information exchange during the measurements with other beacons. This strategy allows to hide the complexity of the multi-vehicle localization. We have tested the algorithm in a simulation and a real experimentation. Both give consistent and precise results.

This work assumes that the robot orientation estimation is sufficiently precise at any time to avoid the linearity problem of the range-only measurements. This issue can be solved by using sensor able to make this first estimation. This 
algorithm can be improved by implementing a solution able to handle this non-linearity and maintains the consistency of the localization in all cases.

\section{ACKNOWLEDGMENT}

This work has been sponsored by the French government research programm Investissements d'avenir through the RobotEx Equipment of Excellence (ANR-10-EQPX-44) and the IMobS3 Laboratory of Excellence (ANR-10-LABX-1601), by the European Union through the programm Regional competitiveness and employment 2007-2013 (ERDF - Auvergne region), by the Auvergne region and by French Institute for Advanced Mechanics

\section{REFERENCES}

[1] S. I. Roumeliotis and G. A. Bekey, "Distributed multirobot localization," IEEE Transactions on Robotics and Automation, vol. 18, no. 5, 2002.

[2] A. Martinelli, "Improving the precision on multi robot localization by using a series of filters hierarchically distributed," in International Conference on Intelligent Robots and Systems (IROS), IEEE, 2007.

[3] L. C. Carrillo-Arce, E. D. Nerurkar, J. L. Gordillo, and S. I. Roumeliotis, "Decentralized multi-robot cooperative localization using covariance intersection," in International Conference on Intelligent Robots and Systems (IROS), IEEE, 2013.

[4] K. Lassoued, O. Stanoi, P. Bonnifait, and I. Fantoni, "Mobile robots cooperation with biased exteroceptive measurements," in International Conference on Control Automation Robotics \& Vision (ICARCV), IEEE, 2014.

[5] N. Karam, F. Chausse, R. Aufrere, and R. Chapuis, "Localization of a group of communicating vehicles by state exchange," in International Conference on Intelligent Robots and Systems (IROS), IEEE, 2006

[6] S. J. Julier and J. K. Uhlmann, "General decentralized data fusion with covariance intersection," in Handbook of Multisensor Data Fusion: Theory and Practice, 2009.

[7] H. Li and F. Nashashibi, "Cooperative multi-vehicle localization using split covariance intersection filter," IEEE Intelligent transportation systems magazine, vol. 5, no. 2, 2013.
[8] T. R. Wanasinghe, G. K. I. Mann, and R. G. Gosine, "Decentralized Cooperative Localization for Heterogeneous Multirobot System Using Split Covariance Intersection Filter," in Canadian Conference on Computer and Robot Vision (CRV), 2014.

[9] K. Lassoued, I. Fantoni, and P. Bonnifait, "Mutual localization and positioning of vehicles sharing GNSS pseudoranges: Sequential bayesian approach and experiments," in International Conference on Intelligent Transportation Systems (ITSC), IEEE, 2015.

[10] A. Howard, M. J. Matark, and G. S. Sukhatme, "Localization for mobile robot teams using maximum likelihood estimation," in International Conference on Intelligent Robots and Systems (IROS), vol. 1, IEEE, 2002.

[11] A. Howard, M. J. Mataric, and G. S. Sukhatme, "Putting the'I'in'team': An ego-centric approach to cooperative localization," in International Conference on Robotics and Automation (ICRA), vol. 1, IEEE, 2003.

[12] A. J. Davison and N. Kita, "Active visual localisation for cooperating inspection robots," in International Conference on Intelligent Robots and Systems (IROS), vol. 3, IEEE, 2000.

[13] J. González, J.-L. Blanco, C. Galindo, A. Ortiz-de-Galisteo, J.-A. Fernández-Madrigal, F. A. Moreno, and J. L. Martinez, "Mobile robot localization based on Ultra-Wide-Band ranging: A particle filter approach," Robotics and autonomous systems, vol. 57, no. 5, 2009.

[14] J. Laneurit, R. Chapuis, and C. Debain, "TRACKBOD, an accurate, robust and low cost system for mobile robot person following," International Conference on Machine Control \& Guidance (MCG), 2016.

[15] H. Li, F. Nashashibi, and M. Yang, "Split covariance intersection filter: Theory and its application to vehicle localization," IEEE Transactions on Intelligent Transportation Systems, vol. 14, no. 4, 2013.

[16] Z. Tao, P. Bonnifait, V. Fremont, and J. Ibanez-Guzman, "Mapping and localization using GPS, lane markings and proprioceptive sensors," in International Conference on Intelligent Robots and Systems (IROS), IEEE, 2013.

[17] Y. Bar-Shalom, X. R. Li, and T. Kirubarajan, Estimation with Applications to Tracking and Navigation: Theory Algorithms and Software. John Wiley \& Sons, 2004.

[18] J. R. Foerster, "The effects of multipath interference on the performance of UWB systems in an indoor wireless channel," in Vehicular Technology Conference (VTC), vol. 2, IEEE, 2001. 